\title{
Recent update on epidural blood patch
}

Received December 1, 2021

Revised December 25, 2021

Accepted December 26, 2021

\section{Corresponding author}

Hwa Yong Shin, M.D., Ph.D., FIPP, CIPS

Department of Anesthesiology and

Pain Medicine, College of Medicine,

Chung-Ang University, 102 Heukseok-

ro, Dongjak-gu, Seoul 06973, Korea

Tel: 82-2-6299-3164

Fax: 82-2-6299-2585

E-mail: pain@cau.ac.kr

\section{Hwa Yong Shin}

Department of Anesthesiology and Pain Medicine, College of Medicine, Chung-Ang University, Seoul, Korea
Epidural blood patch (EBP) is the injection of autologous blood into the epidural space with the intent of sealing off a dural tear and stopping the leakage of cerebrospinal fluid (CSF). EBP may cause an increase in intracranial pressure due to the mass effect of the injected blood volume, causing CSF from the spinal compartment to enter the intracranial compartment. EBP is usually considered in the management of moderate to severe headache $(\mathrm{HA})$ attributed to low CSF pressure, such as post-dural puncture HA (PDPH), CSF fistula HA, and $\mathrm{HA}$ attributed to spontaneous intracranial hypotension $(\mathrm{SIH})$ that does not respond to conservative management. However, prophylactic administration of EBP after accidental dural puncture can hardly be substantiated at present. EBP is generally safe but may rarely be associated with serious complications. Therefore, it should be carefully planned and performed under C-arm fluoroscopic guidance. Although many studies on PDPH and SIH have been conducted until recently, only few reviews have summarized the effectiveness of EBP from the perspective of a pain physician. This article reviews the current literature on the indication, contraindication, procedural consideration, post-procedural management, outcomes, and complications of EBP and the considerations for EBP in patients with COVID-19.

Keywords: COVID-19; Epidural blood patch; Fluoroscopy; Intracranial hypotension; Post-dural puncture headache.

\section{INTRODUCTION}

Epidural blood patch (EBP) is the injection of autologous blood into the epidural space with the intent of sealing off a dural tear and stopping the leakage of cerebrospinal fluid (CSF) [1]. EBP may cause an increase in intracranial pressure (ICP) due to the mass effect of the volume of blood injected, causing CSF from the spinal compartment to enter the intracranial compartment [2].

The first EBP, performed with 2-3 $\mathrm{ml}$ of blood, was reported by Gormley [3] in 1960 after the observation that bloody taps were associated with reduced post-dural puncture headache (PDPH) [4,5]. Since then, the technique of EBP has undergone substantial modifications. In 1980, Crawford reported [6] a success rate of 70\% using 6-15 $\mathrm{ml}$ of blood, compared with $96 \%$ when a larger volume of $20 \mathrm{ml}$ was used [4].

Conservative treatment for the first $24-48 \mathrm{~h}$ is considered the initial management strategy for headache (HA) attributed to low CSF pressure because more than $85 \%$ of such HAs resolve with conservative treatment [7]. Conservative treatment measures include bed rest, intravenous hydration, caffeine supplementation, and analgesics $[7,8]$. EBP is usually considered in the treatment of moderate to severe HA attributed to low CSF pressure that does not respond to conservative management [8]. According to the International Classification of Headache Disorders, 3rd edition (ICHD-3), HA attributed to low CSF pressure is described as orthostatic

This is an Open Access article distributed under the terms of the Creative Commons Attribution Non-Commercial License (http://creativecommons.org/licenses/by-nc/4.0) which permits unrestricted non-commercial use, distribution, and reproduction in any medium, provided the original work is properly cited.

Copyright (C) the Korean Society of Anesthesiologists, 2022 
HA caused by low CSF pressure (either spontaneous or secondary) or CSF leakage, usually accompanied by neck pain, tinnitus, changes in hearing, photophobia, and/or nausea. It remits after normalization of CSF pressure or successful sealing of the CSF leak. Diagnostic criteria are described in Table 1. PDPH, CSF fistula HA, and HA attributed to spontaneous intracranial hypotension (SIH) are subcategories of HA attributed to low CSF pressure.

As pain management is his sub-specialty, the author encounters numerous patients with intractable PDPH and SIH in his practice. Dramatic improvement in such cases is observed with EBP. Although many studies on PDPH and SIH have been conducted until recently, only few reviews have summarized the effectiveness of EBP from the perspective of a pain physician.

Therefore, in this review, the author will summarize the current concepts regarding the indications, mechanisms, technical considerations, outcomes, adverse effects, contraindications, and alternatives to EBP and the peculiarities of EBP during the coronavirus disease 2019 (COVID-19) pandemic.

\section{PATHOPHYSIOLOGY OF HEADACHE ATTRIBUTED TO LOW CSF PRESSURE}

The dura mater is a dense, connective tissue layer made up of collagen and elastic fibers. The classical description of the spinal dura mater is that it is made up of collagen fibers running in a longitudinal direction. However, recent light and electron microscopic studies of human dura mater have contested this classical description [9]. These recent studies describe the dura mater as consisting of collagen fibers arranged in several layers parallel to the surface. Each layer consists of both collagen and elastic fibers that do not demonstrate spe- cific orientation. The outer or epidural surface may indeed have dural fibers arranged in a longitudinal direction, but this pattern is not repeated through successive dural layers. Recent measurements of dural thickness have also demonstrated that the posterior dura varies in thickness and that the thickness of the dura at a particular spinal level is not predictable within an individual or among individuals. Perforation in a thick area of dura may be less likely to lead to CSF leak than perforation in a thin area, and this may explain the unpredictable consequences of dural perforation [9].

The choroid plexus secretes more than $75 \%$ of the CSF; the rest is secreted by the brain capillaries entering the ventricles via the ependyma [5]. The rate of CSF formation, in contrast to its volume, is fairly constant $(0.35 \mathrm{ml}$ per minute or approximately $500 \mathrm{ml}$ per $24 \mathrm{~h}$ in adults) [5,8-10]. CSF is absorbed by arachnoid villi into the cerebral venous sinuses and veins via a valve-like mechanism called bulk flow $[5,10]$. A minor portion of the CSF is absorbed into the cerebral vessels by simple diffusion, and another small portion is likely absorbed via the lymphatics of the cribriform plate region into the nasal submucosa [10]. Old autopsy data estimated the total volume of CSF as approximately $150 \mathrm{ml}$ [10]. In the horizontal position, CSF pressures at the lumbar, cisternal, and presumably intracranial or vertex levels are equal, measuring approximately $65-195 \mathrm{mmH}_{2} \mathrm{O}[10,11]$. On assuming the erect position, the vertex pressure becomes negative, while the lumbar pressure increases to over $400 \mathrm{mmH}_{2} \mathrm{O}$ $[9,11]$. The relationship between CSF pressure and volume is exponential. Withdrawal of approximately $10 \%$ of CSF will decrease the already negative vertex pressure in the upright position by more than $40 \%[5,10]$. If a lumbar dural perforation is so large that CSF leak is greater than CSF production, the CSF pressure will drop. Orthostatic HA is thought to occur if more than $10 \%$ of the total CSF volume is lost $[5,9]$.

Table 1. Diagnostic Criteria for 7.2 Headache Attributed to Low Cerebrospinal Fluid Pressure
A. Any headache* fulfilling criterion $C$
B. Either or both of the following:
1. Low CSF pressure ( $<60 \mathrm{mmH}_{2} \mathrm{O}$ CSF)
2. Evidence of CSF leakage on imaging ${ }^{\dagger}$
C. Headache has developed in temporal relation to the low CSF pressure or CSF leakage, or led to its discovery ${ }^{\ddagger}$
D. Not better accounted for by another ICHD-3 diagnosis

CSF: cerebrospinal fluid, ICHD-3: International Classification of Headache Disorders, 3rd edition, MRI: magnetic resonance imaging, CT: computed tomography. *7.2 Headache attributed to low cerebrospinal fluid pressure is usually but not invariably orthostatic. Headache that significantly worsens soon after sitting upright or standing and/or improves after lying horizontally is likely to be caused by low CSF pressure, although this cannot be relied upon as a diagnostic criterion. ${ }^{\dagger}$ Brain imaging showing brain sagging or pachymeningeal enhancement, or spine imaging (spine MRI, or MRI, CT or digital subtraction myelography) showing extradural CSF. ${ }^{\ddagger}$ Evidence of causation may depend upon onset in temporal relation to the presumed cause, together with exclusion of other diagnoses. 
Two possible explanations for the mechanism of HA due to CSF leak are brain sagging and compensatory dilatation of intracranial veins. First, the lowering of CSF pressure causes traction on the intracranial structures in the upright position. The pain-sensitive nature of these structures leads to the characteristic HA [5]. Second, the loss of CSF produces compensatory venodilatation vis-à-vis the Monro-Kellie doctrine $[1,5,9,11,12]$. The Monro-Kellie doctrine states that the sum of the volumes of the brain, CSF, and intracranial blood is constant. A decrease in CSF volume results in a compensatory increase in blood volume under conditions of unchanged intracranial volume [1,5]. Dilation of the intracranial arteries and veins is a key pathogenetic link in the occurrence and development of HA $[9,12]$. The beneficial effects of vasoconstrictor drugs, such as caffeine and theophylline, support this mechanism [7].

\section{INDICATIONS}

Indications for EBP are PDPH, CSF fistula, SIH, chronic daily HA with postural component, and CSF leak after spinal or thoracic surgery.

\section{Post-dural puncture headache}

PDPH occurs occasionally after spinal anesthesia, myelography, diagnostic lumbar puncture, and accidental dural puncture (ADP) during epidural anesthesia [8,13]. The overall incidence of PDPH after neuraxial procedures varies from $6 \%$ to $36 \%$ [8]. Studies have found that $90 \%$ of these HAs occur within 3 days of the procedure, and $66 \%$ start within the first $48 \mathrm{~h}[5,9,14]$. A postural component may not be present in up to $5 \%$ of cases of PDPH $[4,5]$. Furthermore, in one-third of cases, dural puncture may not have been recognized [4]. Although more than $90 \%$ of PDPHs are self-limiting and resolve spontaneously in 7 to 10 days $[1,14]$, long-term complications of PDPH may include chronic HA, hypoacusis, diplopia, sinus thrombosis, and subdural hematoma $[8,15]$. Moreover, in severe cases, it can cause cerebral herniation and even death if not treated promptly $[1,4,5,16,17]$.

According to the ICHD-3, PDPH was previously called post-lumbar puncture HA and is described as HA occurring within 5 days of a lumbar puncture, caused by CSF leakage through the dural puncture $[4,5,18]$. The diagnostic criteria for PDPH are listed in Table 2. It is usually accompanied by neck stiffness and/or subjective hearing symptoms [18]. However, recent studies indicate that PDPH mostly occurs within 3 days after dural puncture [6-8], and up to $29 \%$ of patients have HA as the only symptom [7]. It remits spontaneously or after sealing of the leak with autologous epidural lumbar patch $[4,5]$. Rarely, the HA may last for months or even years [7,18]. HA is the predominant, but not ubiquitous, presenting complaint. It is described as severe, "searing and spreading like hot metal." The common distribution is over the frontal and occipital areas, radiating to the neck and shoulders $[5,18]$. The pain is exacerbated by head movement and adoption of the upright posture and relieved by lying down [8]. An increase in severity of the HA on standing is the sine qua non of PDPH [9]. Cranial nerve $(\mathrm{CN})$ involvement (vestibulocochlear nerve [CN8] involvement [manifesting as isolated tinnitus, hearing loss, and others] and abducens nerve [CN6] palsy [presenting as diplopia]) can also occur $[1,18]$. Early administration of an EBP within $24 \mathrm{~h}$ of the onset of abducens nerve palsy may be associated with better outcome.

Independent risk factors for PDPH have recently been demonstrated, namely female sex, young age, pregnancy, vaginal delivery, previous history of PDPH, and previous history of chronic HA $[1,5,7,8,14,18]$. Obesity does not increase the risk of PDPH after ADP $[7,13,18]$. Reintroducing the stylet before removing the spinal needle has been recommended to reduce the risk of an arachnoid strand being pulled out through the puncture site and thus reduce the risk of PDPH [5].

The incidence of PDPH has been reported to be associated with needle size, needle orientation, needle design, surgeon skill level, and fatigue. Large spinal needles will clearly produce large dural perforations, with high likelihood of PDPH. Conversely, smaller needles produce smaller dural perforations with a lower incidence of HA $[8,9,14]$. However, fine-gauge spinal needles (29 G or smaller) are technically more difficult to use and, for spinal anesthesia at least, are associated with a high failure rate [9]. A balance must therefore be struck between the risks of PDPH and technical failure; hence, 25 G, 26 G, and 27 G needles probably represent the optimal needle size for spinal anesthesia [9]. There are

Table 2. Diagnostic Criteria for 7.2.1 Post-dural Puncture Headache
A. Headache fulfilling criteria for 7.2 Headache attributed to low cerebrospinal fluid pressure, and criterion $\mathrm{C}$ below
B. Dural puncture has been performed
C. Headache has developed within 5 days of the dural puncture
D. Not better accounted for by another ICHD-3 diagnosis

ICHD-3: International Classification of Headache Disorders, 3rd edition. 
many clinical and laboratory studies that lend credence to the hypothesis that perpendicular orientation of the bevel of a spinal or epidural needle leads to reduction in the incidence of PDPH. The Quincke type spinal needle is reported to be more associated with PDPH than the Whitacre, Sprotte, and Atraucan types [8,9]. Nevertheless, Cochrane Database of Systematic Reviews about the needle tip gauge and tip designs for preventing PDPH reported that the use of traumatic needles was associated with a higher risk of PDPH than the use of atraumatic needles, although large and small gauges showed no significant difference in terms of the risk of PDPH regardless of whether a traumatic or atraumatic needle was used $[8,19]$. ADP during epidural anesthesia is a more common cause of PDPH. During epidural needle placement, ADP occurs at a rate of $1.5 \%$, and around $50 \%$ of these patients develop PDPH $[5,7]$. It has been suggested that the incidence of ADP during epidural anesthesia is inversely related to surgeon experience [5]. However, sleep deprivation, surgeon fatigue, and the effect of night work may be the confounding variables producing the higher incidence of ADP in association with junior personnel performing epidural anesthesia [9].

Imaging techniques for detecting CSF leak, such as brain/ spinal magnetic resonance imaging (MRI), computed tomography (CT) myelography, magnetic resonance (MR) myelography, and radioisotope cisternography, may be helpful. Brain MRI with contrast enhancement may reveal pachymeningeal enhancement, subdural fluid collection, brain sagging, and pituitary hyperemia [4,9]. Diagnostic lumbar puncture may demonstrate low CSF opening pressure or a "dry tap," slightly elevated CSF protein, and elevated CSF lymphocyte count [9].

PDPH should be differentially diagnosed with serious pathology, such as infection, pre-eclampsia, cerebral venous thrombosis, intracranial tumor, intracranial hemorrhage, pituitary apoplexy, uncal herniation, migraine, non-specific HA, and pneumocephalus $[1,5,8,9]$. In case of a HA persisting for a month after a lumbar puncture, despite the use of all therapeutic options, another etiology of HA must be considered [1]. Moreover, PDPH may worsen the clinical course of a previous chronic HA [1].

In the management of PDPH, hydration and bed rest are commonly used for conservative treatment. However, no evidence has been found to suggest that routine bed rest after ADP is beneficial for preventing PDPH. Furthermore, bed rest has been found to likely increase the risk of PDPH compared with early ambulation [5]. There is also no evidence to support the benefit of prophylactic fluid supplementation $[1,5,7]$. Intravenous and parenteral caffeine can however reduce the pain temporarily [5]. Moderate to severe PDPH that has been refractory to conservative measure is a main indication for EBP. Factors that influence the decision to perform EBP are the severity of HA, response to medical treatment, degree of incapacitation by the HA, and nature of the patient's activity. Therapeutic EBP after epidural anesthesia should be delayed until after $24 \mathrm{~h}$ of trying conservative treatment because the high failure rate associated with early administration of EBP has been attributed to interference due to clot formation resulting from the use of local anesthetics [5]. If EBP is to be performed early, it would be better to delay it until the neuraxial blockade has regressed completely, to avoid unpredictable cephalad spread of the local anesthetics due to epidural pressure surge transmitted to the CSF compartment. EBP still remains the gold standard for treatment of PDPH, with complete relief of symptoms in $32 \%$ of cases and partial relief in $73 \%$ [5].

An updated Cochrane review has concluded that there is insufficient evidence to support the performance of prophylactic epidural blood patching (PEBP) $[5,9,14,18]$. Studies in vitro have shown that both lidocaine and CSF have a detrimental effect on coagulation. High concentration of lidocaine causes hypocoagulability and fibrinolysis, whilst CSF has both procoagulant and clot-destabilizing effects [4]. Moreover, PEBP should be performed after full recovery of sensation to prevent accidental total spinal anesthesia [7].

\section{Cerebrospinal fluid fistula headache}

According to the ICHD-3, CSF fistula HA is described as orthostatic HA occurring after a procedure or trauma that causes persistent CSF leakage, resulting in low ICP [1]. It remits after successful sealing of the CSF leak. Diagnostic criteria for CSF fistula HA are listed in Table 3. Evidence of low CSF pressure $\left(<60 \mathrm{mmH}_{2} \mathrm{O}\right)$ and evidence of CSF leakage on CT or MR myelography or radionuclide cisternography are needed to make the diagnosis [1]. EBP may be used to manage CSF fistula that complicates long-term intrathecal catheters inserted for the drainage of CSF after neurosurgical procedures or for the administration of intrathecal medications [20-22].

\section{Spontaneous intracranial hypotension}

SIH is actually a misnomer, as the majority of patients with 
the condition have opening CSF pressures within the normal range. SIH results from non-iatrogenic CSF hypovolemia due to CSF leakage from the spinal canal, rather than CSF hypotension [23-26]. The incidence has been estimated to be around 5/100,000 per year, peaking around the fourth or fifth decade of life and being slightly more common in women (female to male ratio, 3:2) [11,23,25-30]. However, mis- and under-diagnosis of the condition are common, and its actual incidence would be considerably greater $[26,27,29]$. Spontaneous CSF leak is considered a disorder with a variety of clinical manifestations and imaging features, sometimes quite different from what may be seen after dural puncture [10].

According to the ICHD-3, HA attributed to SIH was previously called HA attributed to spontaneous low CSF pressure or primary intracranial hypotension, low CSF-volume HA, and hypoliquorrhoeic HA. It is described as orthostatic HA caused by low CSF pressure of spontaneous origin [24]. It is usually accompanied by neck stiffness, subjective hearing symptoms, nausea, and vomiting [23]. The diagnostic criteria for SIH are listed in Table 4. The diagnosis of SIH cannot be made in a patient who has undergone lumbar puncture in the past month [1].

The most common causes of spontaneous spinal CSF leaks include fragile meningeal diverticula usually associated with nerve root sleeves (42\%), ventral dural tears often caused by calcified disk protrusions or osteophytes (27\%),

Table 3. Diagnostic Criteria for 7.2.2 Cerebrospinal Fluid Fistula Headache
A. Headache fulfilling criteria for 7.2 Headache attributed to low cerebrospinal fluid pressure, and criterion $\mathrm{C}$ below known sometimes to cause persistent CSF leakage (CSF fistula)
B. A procedure has been performed, or trauma has occurred,
C. Headache has developed in temporal relation to the procedure or trauma
D. Not better accounted for by any other ICHD-3 diagnosis

CSF: cerebrospinal fluid, ICHD-3: International Classification of Headache Disorders, 3rd edition. and CSF-venous fistulas (3\%) [24,27]. Heritable connective tissue disorders, such as Marfan syndrome or Ehlers-Danlos syndrome, may predispose patients to the formation of meningeal diverticula $[24,28]$. Therefore, patients with CSF leaks should be screened for connective tissue and vascular abnormalities. Skull-base leaks are rare in SIH [10,11,24].

SIH patients can present with orthostatic HA (92\%), nausea and vomiting (54\%), posterior neck pain (43\%), dizziness (27\%), hypoacusis (28\%), tinnitus (20\%), vertigo (17\%), diminished vision (14\%), photophobia (11\%), and, sometimes, decreased level of consciousness (15\%) [10,11,25,26,28]. Often, the onset of HAs is sudden, and some patients can recall the specific day or even moment when the HAs started [27]. HA is frequently occipital, frontal, or diffuse [26]. On physical examination of SIH patients, maneuvers that increase intra-abdominal pressure will improve their HA or other symptoms. In one study, $8 \%$ of patients had non-orthostatic HA and 3\% did not experience HA [26]. Therefore, not all orthostatic HAs are due to CSF leaks, and not all HAs in people with CSF leaks are orthostatic [25,26]. Moreover, with chronicity, the orthostatic nature of the HA may diminish [10].

The differential diagnosis of SIH may include a primary HA disorder, such as new daily persistent HA, or secondary causes of SIH, including subarachnoid hemorrhage, carotid or vertebral artery dissection, cerebral venous thrombosis, benign intracranial hypertension, post-traumatic HA, and meningitis [25,28]. Postural tachycardia syndrome and cervicogenic HA with postural changes in the neck can be confused with SIH $[25,28]$.

Imaging techniques for detecting the CSF leak are brain/ spinal MRI, CT myelography, MR myelography, and radioisotope cisternography [1]. The best tool for diagnosing SIH is brain MRI with contrast enhancement [23]. MRI may reveal diffuse pachymeningeal enhancement (73\%), venous engorgement (57\%), brain sagging (43\%), subdural fluid collection (35\%), and pituitary hyperemia (38\%) in $\mathrm{SIH}$ $[1,10,11,23,24,26,27]$. Pachymeningeal enhancement is a diffuse meningeal enhancement with gadolinium and a hall-

Table 4. Diagnostic Criteria for 7.2.3 Headache Attributed to Spontaneous Intracranial Hypotension
A. Headache fulfilling criteria for 7.2 Headache attributed to low cerebrospinal fluid pressure, and criterion $\mathrm{C}$ below
B. Absence of a procedure or trauma known to be able to cause CSF leakage*
C. Headache has developed in temporal relation to occurrence of low CSF pressure or CSF leakage, or has led to its discovery ${ }^{\dagger}$
D. Not better accounted for by any other ICHD-3 diagnosis

CSF: cerebrospinal fluid, ICHD-3: International Classification of Headache Disorders, 3rd edition, MRI: magnetic resonance imaging. * 7.2 .3 Headache attributed to spontaneous intracranial hypotension cannot be diagnosed in a patient who has had a dural puncture within the prior month. ${ }^{\dagger}$ Dural puncture to measure CSF pressure directly is not necessary in patients with positive MRI signs of leakage, such as dural enhancement with contrast. 
mark of SIH. The meningeal enhancement involves the pachymeninges but spares the leptomeninges [9,10,23]. Approximately $10 \%$ of patients with SIH have normal brain imaging [24-26]. As the symptoms of SIH persist, there is a notable reduction in MRI findings: dural enhancement and HA symptoms were observed on an average for 15 weeks in one study [28]. Spinal imaging, such as MRI and CT or MR myelography, is an important part of the evaluation of SIH because demonstration of epidural leakage of CSF confirms the diagnosis, and may also direct treatment efforts. In studies for the definitive location of CSF leak on CT or MR myelography, the most common leak location was the thoracic spine (41\%), followed by the cervicothoracic junction (25\%), the cervical spine (14\%), and the lumbar spine (12\%) [26,28]. Leaks were reported to be multiple in $24 \%$ of cases [26]. Radioisotope cisternography is less commonly used currently due to its relative invasiveness, poor spatial resolution, and limited sensitivity and specificity and advances in cross-sectional imaging techniques [24]. The definitive location of the leak may not be established in a significant number of cases investigated with the imaging techniques mentioned above [10]. CT myelography is considered to be the gold standard for the detection of spinal CSF leaks [24,27]. However, because of its risk and invasiveness, brain and spine MRI with enhancement could be performed as first-line investigations in patients with clinical suspicion of SIH [26]. Brain MRI provides a view of the sequelae of a CSF leak and is helpful for initial diagnosis, whereas spinal MRI is used primarily to locate the source of the leak [29].

A CSF opening pressure $<6 \mathrm{cmH}_{2} \mathrm{O}$ during lumbar puncture is considered to be clear evidence of abnormally low CSF pressure and can be used to establish the diagnosis of SIH $[27,28]$. However, it should not be used to exclude SIH. Many patients with SIH have CSF pressures that are in the normal range $\left(6-20 \mathrm{cmH}_{2} \mathrm{O}\right)$, and some may even have symptomatic spinal fluid leaks with CSF pressures in excess of $20 \mathrm{cmH}_{2} \mathrm{O}$ [26-28].

The triad of orthostatic HA, diffuse pachymeningeal enhancement on MRI, and low CSF opening pressure is considered the hallmark for the diagnosis of SIH [10].

Optic nerve sheath diameter measured with transorbital sonography in supine and upright positions in patients with orthostatic HA was found to be significantly decreased, providing a novel non-invasive technique for evaluation of SIH [28,29].

In an evaluation for potential biomarkers, the CSF composition of patients diagnosed with SIH using MRI was com- pared with that of non-SIH patients. Two proteins, lipocalin-type prostaglandin D synthase and brain-type transferrin, had high sensitivity for detecting SIH [28].

Conservative treatment, which includes bed rest, hydration, and caffeine, is often advocated as first-line therapy for patients with SIH [11,27]. In 15-30\% of cases, SIH resolves spontaneously or with only conservative treatment measures within 1-2 weeks from the symptom onset $[11,27,28]$. Medications, such as analgesics, corticosteroids, and theophylline, play only an adjunctive role in the management of SIH [27]. Moderate to severe HA associated with SIH syndrome that has been refractory to conservative measure is the main indication for EBP $[10,30]$. In SIH, the success rate with each EBP varies from $30 \%$ to $90 \%[24,28,30]$. The success rate in one report was $87.1 \%$ after the first targeted EBP and $52 \%$ after the first blind EBP $[24,27,28]$. Many patients require more than one EBP $[28,30]$. A second or even third blood patch may be required at times but should alert the physician to review the differential diagnosis. The success rate of EBP for SIH is significantly less impressive than that seen in PDPH [10,31]. This difference is a consequence of several factors, including the clarity of the level and site of the leak in PDPH and the often more complex anatomy of the leak in SIH.

Complications of SIH are subdural hematoma, rebound intracranial hypertension, cerebral venous sinus thrombosis, superficial siderosis, brachial amyotrophy, and syringomyelia. CSF leaks can recur at variable intervals from previous leaks and with variable frequency; they are not uncommon, although their incidence is not well established [10].

\section{MECHANISM}

Mass effect and epidural plug formation have been suggested as the main mechanisms of action of EBP.

Mass effect theory posits that injection of blood into the epidural space pressurizes the spinal compartment of the subarachnoid space and causes the CSF to be displaced to the more cephalad cranial compartment [23]. Compression of the thecal space for the first $3 \mathrm{~h}$ and presumed elevation of subarachnoid pressure may explain the rapid resolution of the HA [9]. Pressure surge brought about by injection of blood has been demonstrated to be maintained for longer time compared with that caused by injection of crystalloids in experimental models and clinical MRI scans.

Epidural plug theory supposes that the formation of a gelatinous plug, induced by interactions between the injected 
blood and procoagulant components in leaking CSF, seals off the dural tear until the natural healing process restores the integrity of the torn dura. MRI studies have found that epidural blood adheres to the thecal sac, resulting in clot formation for 18-24 $\mathrm{h}$ [7]. At 7-13 h, there is clot resolution, leaving a thick layer of mature clot over the dorsal part of the thecal sac. Animal studies have demonstrated that 7 days after the administration of an EBP, there is widespread fibroblastic activity and collagen formation [9]. That is consistent with the time course of PDPH observed in clinical practice but fails to account for the rapid and often immediate relief upon receiving an EBP.

\section{PROCEDURAL CONSIDERATION}

Before performing an EBP, written informed consent should be obtained from the patient. Information about the EBP, including risks, benefits, and alternative treatment, must be discussed and documented [4]. The patient is positioned prone with a pillow under the chest (for cervicothoracic EBP) or abdomen (for lumbar EBP) [17]. Strict asepsis should be ensured for both collecting blood and accessing the epidural space. Double asepsis with povidone iodine and chlorhexidine-alcohol is usually recommended [17]. The epidural space is first localized with fluoroscopy, and blood is drawn from the patient by an assistant. The use of fluoroscopy promotes localization of the epidural space and is thought to reduce complications [17]. Tuohy needles of 18-20 G are usually used for epidural access.

The needle entry site for epidural access is usually determined by the known or expected location of the dural tear. Studies have shown the mean spread of $15 \mathrm{ml}$ of blood to be six spinal segments cephalad and three segments caudad with a mean volume per segment of $1.6 \mathrm{ml}[4,9]$. Therefore, blood injected during an EBP preferentially spreads in the cephalad direction. For PDPH, the same interspace or one space lower is usually selected $[4,14]$.

EBP should be performed by an experienced pain physician with the administration of contrast agent under real-time fluoroscopy to monitor the spread of the injectate. The reasons for the injection of contrast under real-time fluoroscopy are to confirm that the needle tip is in the epidural space, to detect any intravascular injection, and to therefore predict the spread of the injectate (Fig. 1). Fluoroscopy-guided targeted EBP should be employed if the primary origin of CSF leak has been determined [11,23,25,27]. EBP via an upper thoracic route should be employed if the diagnostic im- aging techniques could not identify the primary origin of CSF leak in cases of SIH because the CSF leaks usually involve the cervicothoracic area. The technique for needle placement in targeted EBP is similar to that used for other epidural injections and most commonly involves either a transforaminal or an interlaminar approach [25]. Needle placement into target dorsal or ventral epidural space and potential spread of EBP are confirmed by contrast epidurogram [25]. Fluoroscopy- or CT-guided targeted transforaminal EBP would be helpful for sealing the ventral surface of the thecal sac, where dural tears caused by disk herniations usually occur in SIH [27].

A blood volume of $20 \mathrm{ml}$ is recommended for EBP via interlaminar approach [8]. Injection should stop before $20 \mathrm{ml}$ has been injected if the procedure is not tolerated by the patient (evidenced by intolerable pain or neurologic deficit) $[5,7,18]$. Volumes of blood larger than $20 \mathrm{ml}$ have been used but appear to offer no additional benefit and may increase the risk of side effects $[4,18]$. EBP volume was a strong predictor of EBP efficacy in both univariate and multivariate analyses, with a volume of $20 \mathrm{ml}$ being most associated with an effective EBP [14,23]. Approximately $5 \mathrm{ml}$ is recommended for EBP via the transforaminal approach [25]. The recommended rate of injection is approximately $5 \mathrm{ml} / \mathrm{min}$ [23]. When the first EBP does not result in clinical benefit, a minimum interval of 5 days is recommended before another EBP to limit the risk of spinal cord compression due to excessive EBP volume [11].

\section{POST-PROCEDURAL MANAGEMENT}

There is insufficient evidence to recommend routine blood culture or administration of antibiotics after performing EBP. Nevertheless, EBP should not be performed if there is systemic infection.

Patients should be positioned in the supine position for 2 $\mathrm{h}$ after an EBP to promote clot formation at the supposed site of dural tear $[1,4,23,29]$. Post-procedurally, patients should avoid intense exercise, Valsalva maneuvers, and long journey for approximately 7 days. They should also avoid constipation by using laxatives. These precautions may reduce the risk of dislodgement of the blood clot covering the dural tear. In addition, patients should be told to avoid twisting and bending, and to keep their backs straight, as these measures are thought to reduce the risk of HA recurrence [4]. 

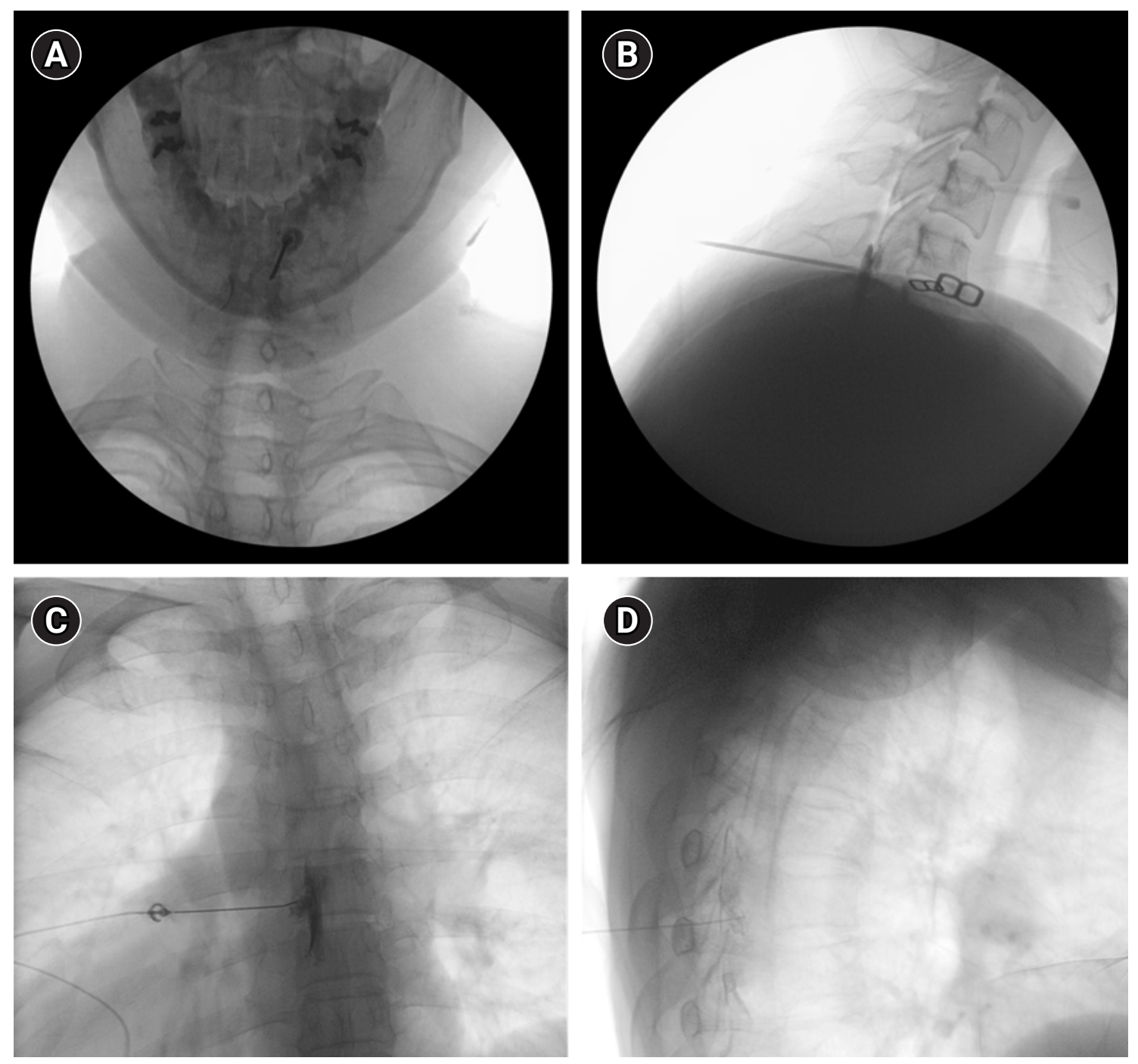

Fig. 1. C-arm fluoroscopy-guided epidural blood patch. Interlaminar approach, cervical: (A) anteroposterior view, (B) lateral view. Transforaminal approach, thoracic: (C) anteroposterior view, (D) lateral view.

\section{OUTCOME}

Estimates for success rates of non-targeted EBP vary widely, with success rates of $30-70 \%$ reported for the initial epidural patch [27]. Targeted EBP may be more effective than blind EBP, with $87 \%$ of targeted EBPs achieving treatment success, compared with $56 \%$ of blind EBPs $[27,29,30]$. In one study, a smaller proportion of patients initially treated with targeted EBP required repeat EBP compared with those initially treated with blind EBP ( $21 \%$ and $61 \%$, respectively) [30]. EBP is considered ineffective or inadequately performed if symptoms do not subside within 2 days of its administration [1]. The initial EBP is reported to be associated with a success rate of approximately $93 \%$ for inducing partial or complete relief of HA [5]. With complete relief as the tar- get, the success rate drops to around $75 \%$ only. The success rates of the first $\mathrm{EBP}$ are $95 \%$ for ADP with spinal needle and $30-75 \%$ for ADP with epidural needle in PDPH. A second EBP may be considered after other causes of HA have been excluded. If the first EBP produced some improvement in symptoms but the HA recurs, a second EBP can be considered. However, in cases where the first EBP has no effect or fails to relieve the symptoms, other causes of HA must be considered, and involvement of other specialties is recommended [4]. Approximately $30 \%$ of patients require a second EBP, following which 50\% experience complete relief, 36$38 \%$ experience partial relief, and $12-14 \%$ experience no relief $[4,5,18]$.

Previous studies reported some factors associated with better outcome after EBP-older age, absence of tinnitus, 
pachymeningeal enhancement, subdural fluid collection, iter $>2 \mathrm{~mm}$ below the incisural line (the iter being defined as the opening of the Sylvian aqueduct and incisural line along the plane extending from the anterior tuberculum sellae through the confluence of the great cerebral vein, inferior sagittal sinus, and straight sinus) on MRI, larger volume of blood ( $>20 \mathrm{ml}$ ), and longer time interval between dural puncture and EBP (more than $48 \mathrm{~h}$ ) [4,23]. Sex and body mass index were found to have no correlation with response to EBP [23].

\section{COMPLICATIONS OF EBP}

Common adverse effects of EBP are HA, backache, neck pain, radicular irritation by blood by-products, and mild pyretic reaction $[11,18]$. These are usually mild and transient. The incidence of backache after EBP is reported as approximately $80 \%$, with resolution by 4 weeks in most cases $[4,18]$. It is thought to be a consequence of increased pressure within the spinal canal resulting from the injection of blood [4]. Delayed radicular symptoms have also been described and may be related to irritation of the nerve roots by hemolytic by-products of the injected blood $[4,18]$.

An important complication experienced by many patients after successful closure of a CSF leak is rebound intracranial hypertension (RIH) $[27,30]$. Caused by an increase in CSF pressure, this phenomenon is characterized by the development of a new HA phenotype shortly after closure of the leak site by EBP or surgery. Most commonly, HAs associated with RIH are worse when lying down and may change in location, compared with SIH. They often occur in the frontal or periorbital region, while occipital HAs are typical for SIH. Symptoms may develop rapidly after EBP or over the course of days to weeks. Treatment is typically with acetazolamide or topiramate [27]. If symptoms are severe, therapeutic lumbar puncture may help relieve them immediately. RIH is very common, although it is not usually severe and may resolve within several days in many cases [27].

Reported rare adverse effects of EBP are chronic adhesive arachnoiditis, subdural or spinal hematoma, seizure, cerebral venous sinus thrombosis, transient bradycardia, infection, intracerebral hemorrhage, facial nerve palsy, visual disturbance, incontinence, monoplegia, cerebral ischemia, cauda equina syndrome, pneumocephalus, formation of a calcific epidural mass, and scarring of the epidural space with distortion of epidural anatomy $[11,18,29,30]$.

The failure rate of EBP is $15-20 \%$. The incidence of dural puncture during an EBP may be less than $1 \%$ but may worsen PDPH by inadvertently creating additional dural rent(s) $[4,14]$. If dural puncture occurs during an EBP, it may increase the risk of intrathecal blood injection, which can lead to an intrathecal or subdural hematoma $[4,32,33]$.

\section{COMPLICATIONS RELATED TO NOT PERFORMING EBP}

Complications related to continued CSF leak if EBP is not performed are chronic HA, chronic back pain, cranial subdural hematoma, cerebral venous sinus thrombosis, cranial nerve palsy (CN 6 and 7), seizures, and, more rarely, death [4].

In one study among parturients, 18 months after delivery, significantly more women in the dural puncture group (28\%) reported HAs, compared with controls (5\%). In the dural puncture group, the HA rate among women who received EBP was $20 \%$, compared with $40 \%$ among those who did not. CSF leak causes caudad shift of the brain, which may lead to rupture of the fragile subdural bridging veins and bleeding into the subdural space. Minimizing CSF leak with an EBP may therefore reduce the incidence of cranial subdural hematoma [24]. Hypercoagulability in pregnancy increases the risk of thrombotic complications. Dural puncture may further increase the risk of cerebral venous sinus thrombosis due to both damage to the cerebral venous endothelium caused by a negative spinal-cranial pressure gradient and stasis from cerebral vasodilation [1].

ADP itself could be associated with reduced quality of future epidural blocks and higher incidence of complications, although EBP administered after ADP does not appear to further increase the risk of future adverse effects [4].

\section{CONTRAINDICATIONS}

Absolute contraindications to EBP are coagulation disorders, infection at the puncture site, febrile illness, bacteremia or septicemia, and patient refusal [15]. Relative contraindications are gross anatomic deformity, acquired immune deficiency syndrome, and leukemia. In several studies, EBP was found safe if the platelet count was more than 75,000 / $\mathrm{mm}^{2}$. However, the author does not recommend EBP when the platelet count is less than $100,000 / \mathrm{mm}^{2}$.

\section{ALTERNATIVES TO EBP}

Initial conservative treatment regimens center on non-in- 
vasive modalities to counteract the proposed mechanisms of HA attributed to low CSF pressure, namely CSF loss and cerebral vasodilation. For example, hydration therapy has been proposed to increase CSF production. Although this modality has remained popular, till date, there is no evidence to support its use [15]. In addition to hydration therapy, other conservative options include bed rest, supine/ prone positioning, and abdominal binders, although these are also not supported by evidence [15].

First-line medications are non-steroidal anti-inflammatory drugs (NSAIDs), acetaminophen, opioids, or combination medications commonly prescribed for tension and migraine HAs (in which barbiturates, acetaminophen, and caffeine are added in one formulation) [15]. Caffeine and theophylline are some of the most studied medications for relief of HA attributed to low CSF pressure. These medications act by two mechanisms. First, they interfere with calcium uptake by the sarcoplasmic reticulum, block phosphodiesterase, and antagonize adenosine, which all result in cerebral vasoconstriction. Second, they increase CSF production by stimulating sodium-potassium pumps [15]. Adrenocorticotropic hormone (ACTH), cosyntropin (an ACTH analogue), and hydrocortisone (which interacts with the ACTH axis) have also been proposed as a therapeutic option. The mechanism by which these medications treat HA attributed to low CSF pressure is unclear, although several theories exist, including expansion of blood volume by releasing aldosterone, dural edema causing overlap of dural hole, increase in CSF production through active sodium transport, and increase in brain $\beta$-endorphins [15]. Pharmacologic therapy with medications that are effective in treating other HA and pain syndromes has also been trialed. Sumatriptan, a serotonin type 1-d receptor agonist, commonly used in treating migraines, showed promise in early case series studies. Methylergonovine, a commonly used uterotonic agent, has also been used to treat migraines because of its alpha activity. Gabapentin and pregabalin have analgesic properties and can inhibit the sympathetic pathway of pain, which may contribute to the pain of HA attributed to low CSF pressure [15].

Pain intervention, such as greater occipital nerve block (GONB) or sphenopalatine ganglion block (SPGB) for the treatment of HA attributed to low CSF pressure showed beneficial effects in reducing pain severity, although the evidence is limited $[7,15,17,18]$. Dural stretching may activate the trigeminal nucleus caudalis, causing some of the pain from HA attributed to low CSF pressure, and this pathway may be blocked by GONB. SPGB is believed to work by blocking parasympathetic outflow to cerebral vasculature, which halts vasodilation [15].

No evidence has been found to support the use of intrathecal catheterization, epidural saline, epidural dextran, epidural hydroxyethyl starch, and epidural/intrathecal/parenteral opioids to treat or prevent PDPH $[1,5,8,13,15,16]$.

Fibrin glue and allogeneic EBP can be other alternatives to EBP. Injection of fibrin glue may be used in cases of persistent HA attributed to low CSF pressure with the identified CSF leak site when repeated EBPs have failed $[16,28,34]$. Allogeneic EBP may be considered if there is concern over the use of autologous blood, as in febrile, HIV-positive, or COVID-19 patients. In such cases, it is appropriate to use crossmatched and tested blood, and the procedure should be conducted under aseptic conditions [17]. Nonetheless, allogeneic EBP has been criticized in view of the risk of transmitting prion diseases, such as Creutzfeldt-Jakob disease $[35,36]$. For patients with CSF leaks that are confidently localized by imaging but in whom epidural patching has failed, surgical closure of the dural perforation may be considered $[4,27]$. Procedures include clipping of the leaking root sleeve, epidural packing, and primary dural repair $[7,23]$. If the site of CSF leak cannot be identified and confidently localized, surgical treatment options are limited [27].

\section{EBP IN PATIENTS WITH COVID-19}

There have been recommendations from several medical societies to maximize the use of regional and neuraxial anesthesia for COVID-19 patients in the perioperative period. This is hypothesized to reduce the risk of aerosolization of viral particles, which is associated with the airway manipulation that occurs with general anesthesia. As a result, the use of neuraxial anesthesia for COVID-19 patients has increased over the past few months. Inevitably, a percentage of these patients may develop complications such as PDPH, requiring an EBP to manage their symptoms. A primary concern regarding the use of EBP in COVID-19 patients is the possibility of seeding the virus in the central nervous system (CNS). In general, an extremely low risk of transferring a blood-borne pathogen into the CNS has been reported when performing EBP with autologous blood for a patient with an ongoing infection. Another important concern is related to the known hypercoagulable state in COVID-19 patients and associated organ dysfunction that may alter the metabolism of anticoagulants [17]. These patients will often be treated with anticoagulants, such as unfractionated hep- 
arin, low-molecular weight heparin, or direct oral anticoagulants. Before performing EBP in this population, laboratory studies and the last dose of anticoagulant must be reviewed. The risks of suspending anticoagulation for an EBP should be carefully weighed against the risks associated with a hypercoagulable state, and it should be discussed with a hematologist [17].

In COVID-19 patients with PDPH, it is important to employ conservative treatments (hydration; bed rest; caffeine; analgesics, such as acetaminophen and NSAIDs; theophylline; and cosyntropin [an ACTH analogue]) and to wait till the resolution of active infection $[5,17]$. If these fail, interventions other than EBP, including bilateral GONB or SPGB, could also be considered [17]. If severe and disabling PDPH persists despite conservative treatments and nerve blocks, EBP should be considered. A fluoroscopic approach may be preferable as it has been shown to be more precise and associated with a higher success rate than a blind technique [17]. The prone position may also offer greater distance for the care team from the patient's face and may be more comfortable for patients with COVID-19 and improve their respiratory mechanics. Complete personal protection for care personnel, including an N95 mask, a face shield, a gown, a head cover, and sterile gloves, must be utilized. The patient should remain masked throughout the procedure [17].

\section{CONCLUSION}

The main indications for EBP are PDPH and SIH. EBP may be considered in cases of moderate to severe $\mathrm{PDPH}$ and $\mathrm{SIH}$ that are refractory to conservative measures. Prophylactic administration of EBP after ADP can hardly be substantiated at present. Before EBP, diagnostic testing, such as brain and spine MRI with enhancement, is essential for both establishing the diagnosis and localizing CSF leaks. EBP is generally safe but may rarely be associated with serious complications. Therefore, strict asepsis should be employed for both blood collection and epidural puncture. Moreover, EBP should be performed in the prone position under C-arm fluoroscopic guidance. Complete personal protection for care personnel, including an N95 mask, a face shield, a gown, a head cover, and sterile gloves, must be utilized when performing EBP in COVID-19 patients. Further investigation, including a large qualified trial on EBP, is warranted, and careful attention should be paid to the timing, prophylactic use, and injection volume and speed of EBP until substantial evidence is available.

\section{FUNDING}

None.

\section{CONFLICTS OF INTEREST}

No potential conflict of interest relevant to this article was reported.

\section{DATA AVAILABILITY STATEMENT}

Data generated or analyzed during this study are included in this published article listed in references.

\section{ORCID}

Hwa Yong Shin, https://orcid.org/0000-0002-8721-3070

\section{REFERENCES}

1. Ljubisavljevic S. Postdural puncture headache as a complication of lumbar puncture: clinical manifestations, pathophysiology, and treatment. Neurol Sci 2020; 41: 3563-8.

2. Fichtner J, Fung C, Z'Graggen W, Raabe A, Beck J. Lack of increase in intracranial pressure after epidural blood patch in spinal cerebrospinal fluid leak. Neurocrit Care 2012; 16: 444-9.

3. Gormley JB. Treatment of postspinal headache. Anesthesiology 1960; 21: 565-6.

4. Russell R, Laxton C, Lucas DN, Niewiarowski J, Scrutton M, Stocks G. Treatment of obstetric post-dural puncture headache. Part 2: epidural blood patch. Int J Obstet Anesth 2019; 38: 104-18.

5. Buddeberg BS, Bandschapp O, Girard T. Post-dural puncture headache. Minerva Anestesiol 2019; 85: 543-53.

6. Crawford JS. Experiences with epidural blood patch. Anaesthesia 1980; 35: 513-5.

7. Kwak KH. Postdural puncture headache. Korean J Anesthesiol 2017; 70: 136-43.

8. Patel R, Urits I, Orhurhu V, Orhurhu MS, Peck J, Ohuabunwa E, et al. A comprehensive update on the treatment and management of postdural puncture headache. Curr Pain Headache Rep 2020; 24: 24.

9. Turnbull DK, Shepherd DB. Post-dural puncture headache: pathogenesis, prevention and treatment. Br J Anaesth 2003; 91: 718-29.

10. Mokri B. Spontaneous intracranial hypotension. Continuum (Minneap Minn) 2015; 21: 1086-108.

11. Ferrante E, Trimboli M, Rubino F. Spontaneous intracranial hy- 
potension: review and expert opinion. Acta Neurol Belg 2020; 120: 9-18.

12. Grant R, Condon B, Hart I, Teasdale GM. Changes in intracranial CSF volume after lumbar puncture and their relationship to postLP headache. J Neurol Neurosurg Psychiatry 1991; 54: 440-2.

13. Bolden N, Gebre E. Accidental dural puncture management: 10year experience at an academic tertiary care center. Reg Anesth Pain Med 2016; 41: 169-74.

14. Tubben RE, Jain S, Murphy PB. Epidural blood patch. In: StatPearls. Edited by Abai B, Abu-Ghosh A, Acharya AB, Acharya U, Adhia SG, Aeby TC, et al.: Treasure Island (FL), StatPearls Publishing. 2021.

15. Katz D, Beilin Y. Review of the alternatives to epidural blood patch for treatment of postdural puncture headache in the parturient. Anesth Analg 2017; 124: 1219-28.

16. Russell R, Laxton C, Lucas DN, Niewiarowski J, Scrutton M, Stocks G. Treatment of obstetric post-dural puncture headache. Part 1: conservative and pharmacological management. Int J Obstet Anesth 2019; 38: 93-103.

17. Scemama P, Farah F, Mann G, Margulis R, Gritsenko K, Shaparin N. Considerations for epidural blood patch and other postdural puncture headache treatments in patients with COVID-19. Pain Physician 2020; 23: S305-10.

18. Gaiser RR. Postdural puncture headache: an evidence-based approach. Anesthesiol Clin 2017; 35: 157-67.

19. Arevalo-Rodriguez I, Muñoz L, Godoy-Casasbuenas N, Ciapponi A, Arevalo JJ, Boogaard S, et al. Needle gauge and tip designs for preventing post-dural puncture headache (PDPH). Cochrane Database Syst Rev 2017; 4: CD010807.

20. Kumar V, Maves T, Barcellos W. Epidural blood patch for treatment of subarachnoid fistula in children. Anaesthesia 1991; 46: 117-8.

21. Chauhan C, Francis GA, Kemeny AA. The avoidance of surgery in the treatment of subarachnoid cutaneous fistula by the use of an epidural blood patch: technical case report. Neurosurgery 1995; 36: 612-3; discussion 613-4.

22. Katz J. Treatment of a subarachnoid-cutaneous fistula with an epidural blood patch. Anesthesiology 1984; 60: 603-4.

23. Signorelli F, Caccavella VM, Giordano M, Ioannoni E, Caricato A, Polli FM, et al. A systematic review and meta-analysis of factors affecting the outcome of the epidural blood patching in spontaneous intracranial hypotension. Neurosurg Rev 2021; 44: 307985.

24. Kranz PG, Gray L, Malinzak MD, Amrhein TJ. Spontaneous intracranial hypotension: pathogenesis, diagnosis, and treatment.
Neuroimaging Clin N Am 2019; 29: 581-94.

25. Amrhein TJ, Kranz PG. Spontaneous intracranial hypotension: imaging in diagnosis and treatment. Radiol Clin North Am 2019; 57: 439-451.

26. D’Antona L, Jaime Merchan MA, Vassiliou A, Watkins LD, Davagnanam I, Toma AK, et al. Clinical presentation, investigation findings, and treatment outcomes of spontaneous intracranial hypotension syndrome: a systematic review and meta-analysis. JAMA Neurol 2021; 78: 329-37.

27. Kranz PG, Malinzak MD, Amrhein TJ, Gray L. Update on the diagnosis and treatment of spontaneous intracranial hypotension. Curr Pain Headache Rep 2017; 21: 37.

28. Upadhyaya P, Ailani J. A review of spontaneous intracranial hypotension. Curr Neurol Neurosci Rep 2019; 19: 22.

29. Davidson B, Nassiri F, Mansouri A, Badhiwala JH, Witiw CD, Shamji MF, et al. Spontaneous intracranial hypotension: a review and introduction of an algorithm for management. World Neurosurg 2017; 101: 343-9.

30. Rettenmaier LA, Park BJ, Holland MT, Hamade YJ, Garg S, Rastogi R, et al. Value of targeted epidural blood patch and management of subdural hematoma in spontaneous intracranial hypotension: case report and review of the literature. World Neurosurg 2017; 97: 27-38.

31. So Y, Park JM, Lee PM, Kim CL, Lee C, Kim JH. Epidural blood patch for the treatment of spontaneous and iatrogenic orthostatic headache. Pain Physician 2016; 19: E1115-22.

32. McInerney HJ, Lee M, Saunders T, Schabel J, Adsumelli RSN. Symptomatic intrathecal hematoma following an epidural blood patch for an obstetric patient with postdural puncture headache: a case report and synthesis of the literature. Case Rep Anesthesiol 2020; 2020: 8925731.

33. Seemiller J, Challagundla S, Taylor T, Zand R. Intrathecal blood injection: a case report of a rare complication of an epidural blood patch. BMC Neurol 2020; 20: 187.

34. Lee YG, Sa M, Oh I, Lim JA, Woo NS, Kim J. Multiple epidural fibrin glue patches in a patient with spontaneous intracranial hypotension - a case report -. Anesth Pain Med 2019; 14: 335-40.

35. Turner M. Epidural blood patch with allogeneic blood for post-dural puncture headache. Int J Obstet Anesth 2006; 15: 261; author reply 261-2.

36. McKenzie A, Manson L. Allogeneic blood for epidural blood patch-not without risk. Int J Obstet Anesth 2006; 15: 260; author reply $61-2$. 